\title{
Igualdad entre mujeres y hombres* Equality Between Women and Men
}

\author{
MariaCaterina La Barbera \\ Universidad Nebrija \\ mlabarbera@nebrija.es
}

Recibido / received: 23/11/2018

Aceptado / accepted: 18/02/2019

DOI: https://doi.org/10.20318/eunomia.2019.4702

\section{Resumen}

Este artículo trata de la igualdad entre mujeres y hombres como principio fundamental del Estado de Derecho. Se ilustran aquí las tres dimensiones interconectadas de la igualdad entre mujeres y hombres contenidas en la Convención para la eliminación de toda forma de discriminación hacia las mujeres (CEDAW). La CEDAW no se limita a considerar la dimensión formal de la igualdad o igualdad en derechos. Apunta a la necesidad de abordar la dimensión material de la igualdad, o igualdad de hecho, e indica las acciones positivas necesarias para ello. La concepción de la igualdad contenida en la CEDAW apela a su dimensión transformativa, es decir, apunta a la eliminación de los estereotipos y las estructuras sociales que perjudican a las mujeres y aspira a transformar la sociedad en su conjunto en términos igualitarios. Siguiendo recomendaciones recientes del Comité CEDAW, se señala finalmente la necesidad de considerar las discriminaciones que sufren las mujeres como el resultado de la intersección de las estructuras de género con otros ejes de desigualdad interconectadas.

\section{Palabras clave}

Igualdad formal, Igualdad de hecho, CEDAW, Igualdad transformativa, Interseccionalidad, cambio social, justicia global.

\begin{abstract}
This article addresses equality between women and men as a fundamental principle of the Rule of Law. The three interconnected dimensions of equality between women and men that are contained in the Convention for the Elimination of All Forms of Discrimination against Women (CEDAW) are here illustrated. CEDAW does not restrain its focus to the formal dimension of equality or equality before the law. It points to the need to address the substantive dimension of equality, or equality de facto, and indicates the positive measures needed to this end. The conception of equality contained in the CEDAW appeals to the transformative dimension of equality, that is to say, it aims to eliminate stereotypes and social structures that harm women and to transform society as a whole in egalitarian terms. Following recent recommendations of CEDAW Committee, the need to consider women's discrimination as the result of the intersection of gender structures with other interconnected axes of inequality is finally pointed out.
\end{abstract}

\section{Keywords}

Formal equality, Substantive equality, CEDAW, Transformative equality, Intersectionality, social change, global justice. 
SUMARIO. 1. La desigualdad entre mujeres y hombres. 2. La dimensión formal de la igualdad o igualdad en derechos. 3. La dimensión material de la igualdad o igualdad de hecho. 4. La dimensión transformativa de la igualdad o igualdad como criterio para el cambio social.

\section{La desigualdad entre mujeres y hombres}

En 1791, Olympe de Gouge (1791) redactó la Declaración de las mujeres y de las ciudadanas para reivindicar los derechos de las mujeres que no se incluyeron en la Declaración del Hombre y del Ciudadano. La reivindicación de la igualdad entre mujeres y hombres como fundamento de los derechos humanos tiene una larga trayectoria histórica, pero el proceso de juridificación de los derechos de las mujeres ha sido muy lento y está aún lejos de haberse completado.

Hasta el comienzo del siglo $\mathrm{XX}$, las mujeres no tenían la titularidad de muchos derechos fundamentales. El derecho al voto de las mujeres se alcanzará en 1893 en Nueva Zelanda, en 1906 en Finlandia, en 1928 en el Reino Unido, en 1931 en España, en 1946 en Italia, en 1971 en Suiza, en 1997 en Qatar, en 2006 en los Emiratos Árabes, en 2015 en Arabia Saudí ${ }^{1}$. El derecho al voto de las mujeres, para el cual luchó el movimiento sufragista, fue solo un primer paso dentro de un proceso de reivindicación de derechos que ha pasado por la igualdad de condiciones laborales, la tutela de la salud sexual y reproductiva y la persecución de la violencia por parte de la pareja. A pesar de las importantes conquistas realizadas ${ }^{2}$, incluso en Europa -donde la igualdad entre mujeres y hombres ha alcanzado los niveles más altos- las mujeres sufren aún muchas discriminaciones por ser mujeres.

Existen datos alarmantes sobre violencia contra las mujeres ${ }^{3}$. Una de cada tres mujeres europeas mayores de 15 años ha sido víctima de violencia física o sexual. Más en detalle: 1 de cada 10 mujeres experimenta alguna forma de violencia sexual; 1 en 20 ha sido violada, y 1 de cada 5 tiene experiencia de violencia física y/o sexual de la mano de su pareja o ex pareja (Fundamental Rights Agency, 2014). La violencia contra las mujeres no es el resultado de actos individuales de mala conducta. Es un problema profundamente arraigado en las relaciones estructurales entre mujeres y hombres que produce tanto desigualdades económicas como de participación en las instituciones públicas y en la toma de decisiones económicas (Naciones Unidas 2006, Walby et al. 2017).

El Índice de la Igualdad de Género 2017, muestra que la distribución de las tareas domésticas sigue siendo desigual en la mayoría de los Estados miembros de la Unión Europea (European Institute for Gender Equality, 2017). En los países miembros, el $78,7 \%$ de las mujeres realiza la mayoría de las tareas domésticas diarias, mientras solo el $27,5 \%$ de las mujeres realiza actividades deportivas, culturales o de ocio fuera del hogar. La distribución desigual de las tareas domésticas se ha identificado como una de las principales causas de la

\footnotetext{
1 Véase Sufragistas [Suffraggettes] dirigida por Sarah Gavron; producida por Aliso Owen, Faye Ward ; escrita por Abi Morgan; director de fotografía, Eduard Grau; música, Alexandre Desplat Carey Mulligan, Helena Bonham Carter, Brendan Gleeson, Anne-Marie Duff, Meryl Streep.

2 En España, por ejemplo, hasta el 1966 las mujeres no tuvieron acceso a la judicatura (Ley 96/1966); hasta 1975 las mujeres casadas no tuvieron capacidad jurídica (Ley 14/1975); hasta 1981 no fueron titulares de la patria potestad compartida ni podían disponer de los bienes gananciales (Ley 11/1981).

3 Véase la voz "Violencia de género" (Marugán Pintos, 2013) en el núm. 4 de esta revista.
} 
discriminación de las mujeres en el mercado laboral, la representación política y la toma de decisiones (Okin, 1989).

Dicho índice muestra también que el acceso al empleo y a buenas condiciones de trabajo todavía no está equilibrado: solo el $39,6 \%$ de las mujeres accede a un empleo a tiempo completo en la Unión Europea (European Institute for Gender Equality, 2017). Los datos en España demuestran que las mujeres tienen más empleo temporal, a tiempo parcial o de baja responsabilidad que los hombres (Instituto Nacional de Estadística, 2017). Es decir, la mayoría de las mujeres tiene ingresos menores que los de sus compañeros (Eurostat, 2018). Aunque el salario base está establecido por ley, sin diferencia entre hombres y mujeres para un mismo puesto de trabajo, los complementos por productividad, horas extra y puestos de dirección revelan diferencias importantes entre hombres y mujeres y explican la existencia de la brecha salarial (European Institute for Gender Equality, 2017).

Además, la vida laboral de las mujeres se interrumpe más frecuentemente y más a menudo salen del mercado laboral, con las relativas repercusiones que ello implica en la cotización a la Seguridad Social y, finalmente, en las pensiones (Ayuso y Chuliá, 2018). Los datos demuestran que estas condiciones laborales son a menudo elegidas para conciliar vida familiar y laboral, ya que tanto las responsabilidades de cuidados de niños y mayores como las tareas domésticas siguen siendo soportadas mayoritariamente por las mujeres (Instituto Nacional de Estadística, 2017). El 44\% de los europeos considera que el rol más importante de las mujeres es el cuidado del hogar y de los niños (Comisión Europea, 2018).

La desigualdad de género sigue siendo importante también en la representación política. La proporción de mujeres ministras en los países de la Unión Europea es del $26,8 \%$ y del $27,7 \%$ la de mujeres parlamentarias. El desequilibrio de género también es relevante en la toma de decisiones económicas. La proporción de mujeres y hombres en los consejos directivos de compañías que cotizan en bolsa y de bancos centrales es aún muy baja. La proporción de mujeres asciende a tan solo al 21,7\% (European Institute for Gender Equality, 2017).

Estos números muestran claramente que incluso en los países que han avanzado más en términos de igualdad, la igualdad efectiva entre mujeres y hombres es muy baja. Pero, además, la situación de desigualdad entre mujeres y hombres es mucho más grave si tenemos en cuenta no solo a las mujeres nacionales de los Estados miembros de la Unión Europea, sino también a las mujeres migrantes. ONU Mujeres (2016) estima que la cifra de las personas que migran para trabajar en el servicio doméstico asciende a nivel global a 11,5 millones. Aproximadamente 1 de cada 5 personas empleadas en el servicio doméstico era migrante en 2013; siendo mujeres el 73.4\%. Según la Organización Internacional del Trabajo, "las trabajadoras de países de bajos ingresos encuentran cada vez más oportunidades de trabajo en el sector doméstico, a menudo en situación de salarios bajos, malas condiciones laborales y escasa protección legal" (Organización Internacional del Trabajo, 2016).

\section{La dimensión formal de la igualdad o igualdad de derechos}

Recalcando la formulación de la Declaración del Hombre y del Ciudadano de 1789, el artículo 1 de la Declaración Universal de los Derechos Humanos de 1948 (en adelante DUDH) proclama que "todos los seres humanos nacen libres e iguales en dignidad y derechos", estableciendo la igualdad en derechos como principio regulativo del Estado de Derecho contemporáneo. Asimismo, el preámbulo afirma que "la libertad, la justicia y la paz en el mundo tienen por base el reconocimiento de 
la dignidad intrínseca y de los derechos iguales e inalienables de todos los miembros de la familia humana". El preámbulo subraya además que "las Naciones Unidas han reafirmado en la Carta su fe en los derechos fundamentales del hombre [sic], en la dignidad y el valor de la persona humana y en la igualdad de derechos de hombres y mujeres; y se han declarado resueltas a promover el progreso social y a elevar el nivel de vida dentro de un concepto más amplio de libertad".

Mientras el artículo 2 DUDH establece el principio de no discriminación "sin distinción alguna de raza, color, sexo, idioma, religión, opinión política o de cualquier otra índole, origen nacional o social, posición económica, nacimiento o cualquier otra condición", el artículo 7 DUDH declara el principio de igualdad formal: "todos son iguales ante la ley y tienen, sin distinción, derecho a igual protección de la ley". A partir del reconocimiento de la intrínseca dignidad de todos los seres humanos contenido en la DUDH, la igualdad y la no discriminación se han convertido en principios regulativos del Estado de Derecho. Es decir, la igualdad no es concebida como un punto de partida, sino una meta al que el Estado de Derecho quiere alcanzar.

El género, la sexualidad, la raza, la (dis)capacidad, el idioma, la religión, la opinión política, el origen nacional y la posición económica son elementos diferenciales entre los seres humanos que marcan el desigual acceso a recursos, opciones, derechos y libertades. Las condiciones personales y sociales que nos diferencian son elementos que definen nuestra situación subjetiva a la vez que se entrelazan con estructuras de marginación y exclusión (La Barbera, 2015). Se trata de diferencias que se insertan en estructuras sociales que colocan de forma sistemática a los blancos, varones, heterosexuales, de formación cristiana y de clase medio-alta en una situación privilegiada (Mackinnon, 1983: 636).

Estas jerarquías incluyen limitaciones en el acceso a derechos y libertades e implican algo más que la injusta distribución de los recursos (Young, 1990; Fraser, 1997). La discriminación estructural hacía las mujeres está vinculada con los desequilibrios de poder dentro y fuera de la familia (Okin, 1989). Consiste en la privación sistemática de las oportunidades para acceder y disfrutar en igualdad de las opciones de vida disponibles.

Si no se remueven los obstáculos sociales que históricamente han impedido el acceso a bienes, recursos y oportunidades, el Derecho sigue perpetuando las desventajas sociales existentes. Sin embargo, la igualdad entendida como principio formal no proporciona soluciones frente a las discriminaciones de hecho. Si usamos la metáfora de quienes compiten en una carrera, si todo el mundo no ha llegado a la línea de partida la carrera no puede ni siquiera empezar. En las famosas palabras del presidente de los Estados Unidos, Lyndon Johnson, "no es suficiente abrir las puertas de las oportunidades. Todos nuestros ciudadanos deben tener la capacidad de atravesar esas puertas" ${ }^{4}$. Para ello, es necesario considerar las condiciones que obstaculizan el igual disfrute de los derechos y abordar la dimensión material (sustantiva o de hecho) de la desigualdad.

Una etapa fundamental en la protección y garantía de los derechos de las mujeres fue marcada por la Convención sobre la eliminación de todas las formas de discriminación contra la mujer ${ }^{5}$ (en adelante CEDAW por su sigla en inglés) aprobada por la Asamblea General de las Naciones Unidas en 1979. La adopción

4 El discurso del Presidente Lyndon B. Johnson de 1965 está disponible en http://www.presidency.ucsb.edu/ws/?pid=27021 (última consulta: 6 de abril de 2018).

5 Nótese que en inglés se usa "women" en plural, mientras la traducción española, revelando el enfoque esencialista predominante, utiliza el singular. 
de una convención internacional específica para proteger los derechos de las mujeres ha transformado la manera de concebir los derechos humanos desde la perspectiva de género (Bunch, 1990). La adopción de la CEDAW no implicaba asumir que las mujeres tienen derechos distintos, sino establecer medidas y actuaciones específicas para tutelar sus derechos humanos considerando sus necesidades biológicas específicas y su situación de discriminación histórica.

El Comité para la Eliminación de la Discriminación contra la Mujer (en adelante el Comité) explica que "las necesidades y experiencias permanentes, determinadas biológicamente, de la mujer deben distinguirse de otras necesidades que pueden ser el resultado de la discriminación pasada y presente cometida contra la mujer por personas concretas, de la ideología de género dominante o de manifestaciones de dicha discriminación en estructuras e instituciones sociales y culturales" (Recomendación General n. 25, Medidas especiales de carácter temporal, párrafo 1 del artículo 4 de la Convención sobre la eliminación de todas las formas de discriminación contra la mujer, §11).

A causas de la discriminación histórica sufrida, las mujeres necesitan una especial protección por parte de los Estados para gozar en igualdad de condiciones de los derechos reconocidos a todos los seres humanos. La CEDAW proporciona una hoja de ruta detallada para alcanzar la igualdad entre mujeres y hombres que incluye: la eliminación de los obstáculos legales que impiden el acceso a derechos y libertades en igualdad de condiciones a mujeres y hombres; el reconocimiento de las necesidades específicas de las mujeres tutelando sus derechos sexuales y reproductivos; la eliminación de estereotipos que perpetúan la discriminación de las mujeres; la adopción de acciones positivas para compensar la discriminación histórica y permitir el acceso a las instituciones dónde tradicionalmente las mujeres han sido excluidas de iure o de facto, y la transformación de la sociedad a través de la educación de niños y niñas con perspectiva de género.

En conformidad con la DUDH, la CEDAW establece que "los Estados signatarios están obligados a condenar la discriminación contra las mujeres y adoptar medidas para eliminarla" (art. 2 CEDAW). Al ratificar la CEDAW, los Estados se obligan a garantizar la ausencia de discriminación directa e indirecta contra las mujeres en su legislación, jurisprudencia y praxis administrativa. Es decir, la CEDAW requiere revisar las legislaciones nacionales con el fin de eliminar tanto las normas que prevén un trato abiertamente desigual para las mujeres (discriminación directa) como aquellas que se basan en criterios aparentemente neutros que las perjudican de hecho (discriminación indirecta). Cualquier contrato u otro acuerdo privado que limite la capacidad jurídica de las mujeres "se considerará nulo" (art. 15 CEDAW). Finalmente, la CEDAW requiere que se garantice la tutela por parte de tribunales competentes contra acciones u omisiones discriminatorias cometidas tanto por autoridades públicas (por ej. jueces o policías) como por organizaciones privadas, empresas o particulares.

\section{La dimensión material de la igualdad o igualdad de hecho}

La CEDAW se basa en una conceptualización del género como construcción social injusta (Scott, 1986) que, a partir de las diferencias biológicas entre mujeres y hombres, implica la subordinación jurídica y social de la mitad de la población del planeta. La CEDAW reconoce además que la distinción entre público y privado es ficticia (Pateman, 1983; Olsen, 1985) y que para alcanzar la igualdad entre hombres y mujeres es necesario que los Estados intervengan en ámbitos tradicionalmente considerados privados como los cuidados y eliminen "los patrones culturales que definen el ámbito público como un mundo masculino y la esfera doméstica como el 
dominio de la mujer" (Introducción CEDAW). Estableciendo la obligación de eliminar prejuicios y prácticas sexistas, la CEDAW reconoce la interconexión de tres dimensiones fundamentales de la justicia. La justicia no es concebida solamente como redistribución de los recursos (Rawls, 1979), sino también como reconocimiento de las características específicas invisibilizadas y menospreciadas (Honneth, 1997) e incluye también la representación política (Fraser, 2003). La CEDAW parte de una visión crítica del Derecho y reconoce: que el sistema jurídico puede reproducir la desigualdad; que alcanzar la igualdad requiere alterar el status quo injusto, y que es necesario transformar las estructuras sociales y los procesos políticos que obstaculizan de hecho la igualdad de las mujeres.

La CEDAW considera la dimensión material de la igualdad tomando en consideración las diferencias y desventajas en el punto de partida que impiden de hecho la igualdad en dignidad y derechos de las mujeres. Esto implica considerar la discriminación no tanto como una cuestión individual sino más bien estructural (Young, 2009). Es decir, la CEDAW requiere abordar las estructuras sociales y políticas que generan una exposición sistemática a la discriminación de las mujeres como grupo históricamente desfavorecido. Uno de los avances clave representados por la CEDAW es el foco, expresamente asimétrico, en el grupo que sufre desventajas y la previsión de acciones positivas dirigidas a minimizar la discriminación o compensar las desventajas resultantes de actitudes, comportamientos y estructuras sociales sexistas. Se trata de obligaciones positivas que los Estados han asumido al ratificar la Convención (Recomendación General $n$. 25: § 24).

Las así llamadas "cuotas" son el ejemplo más conocido de acciones positivas. En España, la Ley de Igualdad (L. 3/2007, de 22 de marzo) anima a los poderes públicos a adoptar acciones positivas "a favor de las mujeres para corregir situaciones patentes de desigualdad de hecho respecto de los hombres" (Art. 11, L. 3/2007). La Ley de igualdad establece que en las listas electorales cada sexo no deberá superar el $60 \%$ y no ser inferior al $40 \%$ de cada en cada tramo de 5 candidaturas. Prevé además la "presencia o composición equilibrada" (no más del $60 \%$, no menos del $40 \%$ ) para los nombramientos de cargos directivos de la administración general del Estado, órganos de selección del personal y consejos de administración de empresas con participación pública (disposición adicional primera).

Se trata de medidas provisionales para conseguir que tanto las mujeres como los hombres estén representados y participen en los procesos de toma de decisión política y económica. Ya que los que ocupan posiciones de poder tienden a seleccionar los candidatos entre los que están en sus círculos (los así llamados old boys' network), las cuotas obligan a los partidos y los empleadores a reclutar a más mujeres como grupo históricamente no representado. En caso de méritos comparables, las cuotas sirven para incluir las mujeres y compensar situaciones de discriminación históricas (Bacchi, 2006). El Comité ha aclarado que la aplicación de medidas especiales de carácter temporal es un medio para hacer realidad la igualdad material y no una excepción a las normas de no discriminación e igualdad (Recomendación General n. 25: §14). Es decir, es conceptualmente equivocado definir las cuotas como medidas de "discriminación inversa".

4. La dimensión transformativa de la igualdad o igualdad como criterio para el cambio social

Las acciones positivas como las cuotas alteran los resultados, pero no implican un reexamen en profundidad de las estructuras socio-jurídicas que perpetúan la 
discriminación (Fredman, 2016: 722). Las cuotas atacan los síntomas, pero no las causas de la desigualdad. No ofrecen, por ejemplo, soluciones para las mujeres que, por haber asumido todas las responsabilidades de cuidado familiar, no tienen "méritos comprables". Las cuotas permiten el acceso solo a aquellas mujeres que han alcanzado dichos méritos, sin abordar la raíz de la discriminación, y por lo tanto no son suficientes.

La CEDAW va más allá y requiere alterar las causas de la discriminación hacia las mujeres, transformando la sociedad en su conjunto. La CEDAW afirma que para garantizar la igualdad, los Estados deben eliminar toda forma de discriminación directa, corregir las desventajas de partida a través de medidas especiales, eliminar la violencia, garantizar la "voz" compensando la exclusión política y social, y contrarrestar prejuicios, estigmatización y estereotipos (Fredman, 2016: 727). El objetivo de la CEDAW de erradicar las estructuras que sistemáticamente producen discriminación implica reconocer la igualdad como proyecto transformador de la sociedad en su conjunto (Cook y Cusack, 2011; Laporta, 2016). Los Estados deben "transformar realmente las oportunidades, las instituciones y los sistemas de modo que dejen de basarse en pautas de vida y paradigmas de poder masculinos determinados históricamente" (Recomendación General n. 25: §10).

La CEDAW obliga a los Estados signatarios a modificar los patrones sociales y culturales que determinan prejuicios y prácticas basados en estereotipos sexistas (Art. 5 CEDAW). Superando la idea de igualdad meramente formal, la CEDAW conecta la igualdad material (Art. 4 CEDAW) con su vertiente transformativa (Fredman, 2003: 115). Los Estados signatarios tienen la obligación de hacer frente a la persistencia de estereotipos basados en el género que afectan a las mujeres no sólo a través de actos individuales, sino también a través de la legislación, las instituciones políticas y las estructuras sociales (Recomendación General n. 25: § 7 y 10). Es necesario modificar pues los marcos de referencia, el lenguaje y las representaciones a través de la educación y la formación continua así como las estructuras familiares a través de políticas de conciliación familiar. Pero también, hay que cambiar la manera de enmarcar el problema de la discriminación hacia las mujeres, dejando por ejemplo de representarlo como infrarrepresentación de las mujeres y poniendo en evidencia la sobrerrepresentación de los hombres (Bacchi, 2006).

\section{Conclusiones}

Aunque la CEDAW haya establecido hace 40 años una hoja de ruta muy clara para lograr el cambio socio-jurídico y alcanzar la igualdad, las desigualdades entre mujeres y hombres persisten y se reproducen (Foro Económico Mundial, 2017). Uno de los problemas de las políticas para la erradicación de la discriminación hacia las mujeres ha sido su enfoque "unitario" (Hancock, 2007: 64) que considera el género como la única causa de discriminación hacia las mujeres. Recientemente, sin embargo, el Comité ha reconocido que "las mujeres nunca son solo mujeres" y que existen entre ellas "diferencias que hacen la diferencia" (Crenshaw, 2002: 173) a la hora de sufrir la discriminación de género.

El Comité reconoce que la interseccionalidad (Crenshaw, 1989) ${ }^{6}$ es un concepto clave para comprender el alcance de las obligaciones internacionales de los Estados signatarios. Las discriminaciones que sufren las mujeres por razón de género están interconectadas de manera inseparable con otros factores como la raza, el origen étnico, el origen nacional, la religión o las creencias, la salud, la

\footnotetext{
${ }^{6}$ Véase la voz "Interseccionalidad" (La Barbera, 2017) en el núm. 12 de esta revista.
} 
posición socioeconómica, la edad, la clase, la casta y la orientación sexual. Algunos grupos de mujeres están expuestas a situaciones de vulnerabilidad específica que les afectan de manera única y desproporcionada en comparación con otras mujeres (Crenshaw, 2000).

Las situaciones de vulnerabilidad específica que se generan por la intersección entre ejes de desigualdad interconectados son especialmente complejas en la era de las migraciones fronterizadas (La Barbera, 2019). La interseccionalidad permite abordar las discriminaciones que afectan a las mujeres migrantes como el resultado de la interacción entre los sistemas normativos, económicos y socio-culturales de los países de origen y de destino (La Barbera, 2015). Permite reconocer, por ejemplo, que las mujeres migrantes se encuentran en situación de especial vulnerabilidad porque el mercado laboral en el país de destino no está solo segregado en base al género sino está también racializado (Moré Corral, 2017). Su discriminación en el mercado laboral en el país de destino no se puede abordar adecuadamente si no se reconoce que los distintos ejes de desigualdad operan de forma interconectada.

Los Estados partes deben reconocer y prohibir estas formas de discriminación entrecruzadas y su impacto negativo combinado en la vida de las mujeres (Recomendación General n. 28 relativa a las obligaciones básicas de los Estados partes de conformidad con el artículo 2 de la Convención sobre la eliminación de todas las formas de discriminación contra la mujer, § 18). La interseccionalidad es una categoría irrenunciable para alcanzar la igualdad entre mujeres y hombres.

\section{Bibliografía}

AYUSO, M. y CHULIÁ, E. (2018), ¿Hacia la progresiva reducción de la brecha de género en las pensiones contributivas?, Documento de Trabajo 22/2018, Instituto BBVA, disponible en la siguiente dirección electrónica: https://www.jubilaciondefuturo.es/recursos/doc/pensiones/20160609/esfe/laspensiones-y-la-brecha-de-genero.pdf (fecha de consulta: noviembre de 2018).

BACCHI, C. (2006), "Arguing for and against quotas". En: DAHLERUP, D. (Coord.), Women, Quotas and Politics, London, Routledge, pp. 32-51.

BUNCH, C. (1990), "Women's rights as human rights: Toward a re-vision of human rights", Human Rights Quarterly, 12, pp. 486-498.

COMISIÓN EUROPEA (2018), 2018 Report on equality between women and men in the EU, Luxembourg, Publications Office of the European Union.

COOK, R. y CUSACK, S. (2011), Gender Stereotyping, University of Pennsylvania Press, Philadelphia.

CRENSHAW, K. (1989), "Demarginalizing the Intersection of Race and Sex", University of Chicago Legal Forum, pp. 139-167.

CRENSHAW, K. (2002), "Gender-related aspects of race discrimination, background paper for the United Nations EXPERT MEETING on Gender and Racial Discrimination", Revista de Estudos Feministas, 10, pp. 171-188.

DE GOUGES, O. (1791), Déclaration des droits de la femme et de la citoyenne, République des Lettres, Paris, 2012.

EUROPEAN INSTITUTE FOR GENDER EQUALITY (2017), Gender Equality Index 2017, disponible en la siguiente dirección electrónica: https://eige.europa.eu/rdc/eige-publications/gender-equality-index-2017measuring-gender-equality-european-union-2005-2015-report (fecha de consulta: noviembre de 2018). 
EUROSTAT (2018), Gender pay gap statistics, disponible en la siguiente dirección electrónica: https://ec.europa.eu/eurostat/statisticsexplained/index.php/Gender pay gap statistics\#Gender pay gap levels var y significantly across EU (fecha de consulta: noviembre de 2018).

FORO ECONÓMICO MUNDIAL (2017), The Global Gender Gap Report, World Economic Forum, Ginebra.

FRASER, N. (1997), Justicia interrupta. Reflexiones críticas desde la posición "postsocialista», Universidad de los Andes: Siglo del Hombre Editores, Bogotá.

FRASER, N. (2003), "Social Justice in the Age of Identity Politics: Redistribution, Recognition, and Participation". En: FRASER, N. y HONNETH, A. (Coords.), Redistribution or Recognition: A political-philosophical exchange, Londres, Verso, pp. 7-109.

FREDMAN, S. (2003), "Beyond the Dichotomy of Formal and Substantive Equality: Towards a New Definition of Equal Rights". En: Boerefijn, I. et al. (Coords.), Temporary Special Measures: Accelerating de facto Equality of Women under Article 4(1) UN Convention on the Elimination of All Forms of Discrimination against Women, Oxford, Intersentia, pp. 111-115.

FREDMAN, S. (2016), "Substantive Equality Revisited", International Journal of Constitutional Law, 14 (3), pp. 712-738.

FUNDAMENTAL RIGHTS AGENCY (2014), Violence against women: an EU-wide survey, disponible en la siguiente dirección electrónica: http://fra.europa.eu/en/publication/2014/violence-against-women-eu-widesurvey-main-results-report (fecha de consulta: noviembre de 2018).

HANCOCK, A. (2007), “When multiplication doesn't equal quick addition: Examining intersectionality as a research paradigm", Perspectives on Politics, 5 (1), pp. 63-79.

HONNETH, A. (1997), La lucha por el reconocimiento: por una gramática moral de Ios conflictos sociales, Crítica, Barcelona.

INSTITUTO NACIONAL DE ESTADÍSTICA (2017), Mujeres y hombres en España, Instituto Nacional de Estadística, Madrid.

LA BARBERA, MC. (2015), "Identity and Migration: An Introduction". En: LA BARBERA, MC, Identity and Migration in Europe: Multidisciplinary Perspectives, Dordrecht, Springer, pp. 1-13.

LA BARBERA, MC. (2017), "Interseccionalidad", Eunomía: Revista en Cultura de la Legalidad, 12, pp. 191-198.

LA BARBERA, MC. (2019), "Toward Global Justice: Intersecting Structural Vulnerabilities as a Key Category for Equality Policies in the Age of Bordered Migrations". En: J.C. VELASCO, JC. y La BARBERA MC. (Coords.), Challenging the Borders of Justice in the Age of Migrations, Dordrecht, Springer, pp. 205-224.

LAPORTA HERNÁNDEZ, E. (2016), "Desde la Convención sobre la Eliminación de todas las Formas de Discriminación de la Mujer a la igualdad transformativa en España". En: LA BARBERA MC. Y CRUELLS LÓPEZ, M. (Coords.), Igualdad de género y no discriminación en España, evolución problemas y perspectivas, Madrid, Centro de Estudios Políticos y Constitucionales, pp. 59-79.

MACKINNON, C. (1983), "Feminism, Marxism, Method and State: Toward Feminist Jurisprudence", Signs, 8 (4), pp. 635-658.

MARUGÁN PINTOS, B. (2013), "Violencia de género", Eunomía: Revista en Cultura de la Legalidad, 4, pp. 226-233.

MORÉ CORRAL, P. (2017), Migraciones y trabajo con personas mayores en las grandes ciudades, Centro de Investigaciones Sociológicas, Madrid.

NACIONES UNIDAS (2006), Ending violence against women, UN Publication, Ginebra.

OKIN, S. (1989), Justice, Gender, and the Family, Basic Books, New York. 
OLSEN, F. (1985), "The Myth of State Intervention in the Family", University of Michigan Journal of Law Reform, 18 (4), pp. 835-864.

ORGANIZACIÓN INTERNACIONAL DEL TRABAJO (2016), Migrant Domestic Workers, International Labour Office, Geneva.

PATEMAN, C. (1983), "Feminist critiques of the public/private dichotomy". En: S. BENN y G. GAUS (Coords.), Public and private in social life, New York, St. Martin's Press, pp. 281-303.

RAWLS, J. (1979 [1971]), Una teoría de la justicia, Fondo de Cultura Económica, México.

SCOTT, J. (1986), "Gender: A Useful Category of Historical Analysis", American Historical Review, 91(5), pp. 1053-1075.

WALBY, S. et al. (2017), The concept and measurement of violence against women and men, Policy Press, Oxford.

YOUNG, I. (1990), Justice and the Politics of Difference, Princeton University Press, Princeton.

YOUNG, I. (2009), "Structural Injustice and the Politics of Difference". En: E. GRABHAM, E. et al. (Coords.), Beyond Intersectionality, New York, Routledge, pp. 273-299. 